\title{
ACL Reconstruction with Autologous Hamstring Tendon: Comparison of Short Term Clinical Results between Rigid-fix and PINN-ACL Cross Pin
}

\author{
Seung-Suk Seo, $\mathrm{MD}^{1}$, Chang-Wan Kim, $\mathrm{MD}^{1}$, Tae-Seok Nam, $\mathrm{MD}^{2}$ and Sang-Yeong Choi, $\mathrm{MD}^{1}$ \\ Department of Orthopedic Surgery, ${ }^{1}$ Busan Paik Hospital, University of Inje College of Medicine, Busan; ${ }^{2}$ Nanoori Hospital, Seoul, Korea
}

Purpose: To compare the short term clinical results of anterior cruciate ligament (ACL) reconstruction with autologous hamstring tendon between Rigid-fix and PINN-ACL Cross Pin for femoral side fixation.

Materials and Methods: 127 patients who underwent arthroscopic ACL reconstruction using autologous hamstring tendon and had been followedup for over than one year were enrolled for the present study. Rigid-fix was used in 71 cases (group 1), and PINN-ACL Cross Pin was used in 56 cases (group 2). Clinical and radiological results, operation time, and perioperative complications were compared amongst the two groups.

Results: The International Knee Documentation Committee subjective score and Lysholm score were 94 and 95 in group 1 and 87 and 91 in group 2 , with no statistical difference $(\mathrm{p}=0.892, \mathrm{p}=0.833)$, respectively. However, significant difference was observed in one-leg hop test between the two groups ( $\mathrm{p}=0.032$ ). Five cases in group 1 and 40 cases in group 2 were found to be associated with perioperative complications with statistical difference $(\mathrm{p}<0.0001)$.

Conclusions: There was no resultant difference between the employment of PINN-ACL Cross Pin and Rigid-fix as femoral graft fixation for ACL reconstruction with hamstring tendon. However, PINN-ACL Cross Pin led to complications with extensive operation times. Hence, it needs further improvement of tools for minimization of complications.

Key words: Anterior cruciate ligament reconstruction, Hamstring tendon, Rigid-fix, PINN-ACL Cross Pin.

\section{Introduction}

In anterior cruciate ligament (ACL) reconstruction, hamstring tendon grafts are replacing the popular bone-patellar tendonbone grafts that have been associated with femorotibial joint pain, loss of extension, and patellar tendon rupture ${ }^{1-4)}$. However,

Received May 3, 2011; Revised (1st) June 9, 2011; (2nd) July 15, 2011; Accepted July 27, 2011.

Correspondence to: Chang-Wan Kim, MD.

Department of Orthopedic Surgery, Busan Paik Hospital, University of Inje College of Medicine,

Gaegeum-dong, Busanjin-gu, Busan 633-165, Korea.

Tel: +82-51-890-6257, Fax: +82-51-892-6619

Email: kcw8737@empas.com

This is an Open Access article distributed under the terms of the Creative Common Attribution Non-Commercial License (http://creativecommons.org/licenses/by-nc/3.0/) which permits unrestricted non-commercial use, distribution, and reproduction in any medium, provided the original work is properly cited.

Copyright $\odot$ 2011. KOREAN KNEE SOCIETY

www.jksrr.org tendon-to-bone fixation using hamstring tendon grafts allows for less fixation strength when compared to bone-to-bone fixation using bone-patellar tendon-bone grafts and is not conducive to early rehabilitation as revealed by an animal study, where complete union was obtained $\geq 24$ weeks after $\mathrm{ACL}^{5-7)}$. Therefore, secure graft fixation appears to be crucial for early weight-bearing and aggressive rehabilitation after ACL reconstruction using hamstring tendon grafts.

Currently, a variety of methods for femoral fixation of tendon grafts have been introduced and employed in clinical settings. Biomechanical advantages and disadvantages of individual methods have been well documented in previous studies and new fixation methods are continuously being suggested. Expansion fixation that is based on press-fit fixation of a graft into the femoral tunnel by employing $\geq 1$ cross pin has been reported to render satisfying results in biomechanical and clinical studies ${ }^{8)}$. Cancellous suspension fixation allows the least elongation and accordingly provides the greatest fixation strength and stability amongst various available methods and has the lowest cyclic amplitude ${ }^{9)}$.

In this study, we compared $\geq 1$-year short-term clinical results of 
ACL reconstructions using an expansion fixation device, Rigidfix (Mitek, Norwood, MA, USA) and a cancellous suspension device, PINN-ACL Cross Pin (CondMed Linvatec, Largo, FL, USA).

\section{Materials and Methods}

\section{Materials}

Arthroscopic ACL reconstruction using an autologous quadrupled hamstring tendon graft was performed with Rigid-fix in 87 patients between January 2006 and December 2007 and with PINN ACL Cross Pin in 64 patients between January 2008 and February 2010 at our institution. Of them, 71 patients in whom Rigid-fix was used (group I) and 56 patients in whom PINN ACL Cross Pin was used (group II) were enrolled in this study. The minimum follow-up period was 1 year.

\section{Combined Injury}

The most common combined injury was a meniscus tear (37 cases in group I and 36 cases in group II) followed by medial collateral ligament injury (21 cases in group I and 23 cases in group II) and chondromalacia (36 cases in group I and 29 cases in group II). For the meniscus tears, suture repair and partial menisectomy were performed in 24 cases and 13 cases, respectively, in group I and in 21 cases and 15 cases, respectively, in group II. For the medial collateral ligament injuries, full range of motion was obtained with conservative treatment before ACL reconstruction. Regarding the chondral lesions, patients with minor chondromalacia that could be treated with debridement were included in this study and severe cases that required surgical treatment were excluded.

\section{Surgical Technique}

Transtibial single-bundle ACL reconstruction was performed in all the patients. For the Rigid-fix system, a 3.3-mm cross-pin was passed through the tendon graft in the femoral tunnel and fixed to the femur. For tibial fixation, the graft was fixed by employing Intrafix (Mitek, Norwood, MA, USA) within the tibial tunnel under a tension of $9.07 \mathrm{~kg}$ (20 pounds) with the knee at $20^{\circ}$ of flexion. Subsequently, post-tie fixation was additionally done using a $4.0 \mathrm{~mm}$ Arbeitsgemeinschaft für Osteosynthesefragen (AO) cortical screw in the cortical bone of the anteromedial aspect of the proximal tibia.

For the PINN-ACL Cross Pin system, the quadrupled tendon graft was passed through the graft harness and the terminals were tied together. The graft harness was inserted into the femoral tunnel and an arthroscope was advanced through the transverse tunnel to make sure that the eyelet of the graft harness was at the center of the transverse tunnel. Next, a cross-pin was laterally inserted to pass through the eyelet of the graft harness and the head of the cross-pin was fixed to the surface of the cortical bone of the lateral condyle of the femur. For tibial fixation, tension of $80 \mathrm{~N}$ was maintained on the graft using SE graft tensioner with the knee at $20^{\circ}$ of flexion. Intrafix and a $4.0 \mathrm{~mm} \mathrm{AO}$ cortical screw were used to complete the fixation in the tibial tunnel.

\section{Rehabilitation}

From the first postoperative day, patients began $0^{\circ}-30^{\circ}$ of flexion exercises using a continuous passive motion machine and partial weight-bearing with a crutch and a knee brace was permitted. The range of motion was gradually increased from the $3 \mathrm{rd}$ postoperative week to $\geq 90^{\circ}$ of flexion at the 6th postoperative week and ambulation without an assistive device was permitted. From the 12th postoperative week, patients were allowed to resume light activities of daily living. From the 6th postoperative month, light sports activities including jogging, swimming, and cycling were permitted. Return to previous sport activities or contact sports were allowed after 10 months of surgery when the muscle strength and proprioception were restored. The rehabilitation program was identical for all the patients with meniscal repair.

\section{Clinical and Radiological Assessments}

The operation time was measured based on the tourniquet time. For clinical assessment, the International Knee Documentation Committee (IKDC) subjective scores and Lysholm scores, pivot shift test results, and one-leg hop test results were obtained preoperatively and compared with the ones obtained at the last follow-up. Laxity was evaluated with a KT-1000 arthrometer (Medmetric Corp., San Diego, CA, USA) and anterior displacement was measured with a Telos arthometer (Telos stress device; Austin \& Associate, Inc., Polston, MD, USA) both, before and after the surgery. The pivot shift test results were classified into 4 grades: grade 0 , no side-to-side difference; grade I, slight subluxation or slippage; grade II, definite subluxation; and grade III, subluxation and locking. Regarding the one-leg hop test, the longest distance in 3 consecutive trials was registered and classified into 4 grades based on the comparison with the contralateral side: grade A, $100-75 \%$ of the unaffected side; grade B, $75-50 \%$; grade C, $50-25 \%$; and grade D, $\leq 25 \%$. 


\section{Statistical Analysis}

Statistical analysis was performed using SPSS software (ver. 13.0, SPSS Inc., Chicago, IL, USA). One-leg hop test results and pivot shift test results were analyzed with the chi square test and other variables were analyzed with analysis of variance test and the $t$-test. A value of $\mathrm{p} \leq 0.05$ was considered statistically significant.

\section{Results}

The mean age of the patients was 31.7 years (range, 18 to 46 years) in group I and 32.5 years (range, 19 to 44 years) in group II. The male-to-female ratio was 57/14 in group I and 39/17 in group II. The mean follow-up period was 22.7 months (range, 12 to 37 months) in group I and 14.5 months (range, 12 to 25 months) in group II. The cause of injury was sports injury in 58, traffic accident in 9, and activities of daily living in 4 patients in group I and sports injury in 41 , traffic accident in 10 , and other causes in 5 patients in group II (Table 1).

Table 1. The Patient Demographics

\begin{tabular}{lcc}
\hline & $\begin{array}{c}\text { Group 1 } \\
\text { (Rigid-fix) }\end{array}$ & $\begin{array}{c}\text { Group 2 } \\
\text { (PINN-ACL) }\end{array}$ \\
\hline Mean age (y) & 31.7 & 32.5 \\
Sex (male/female) & $57 / 14$ & $39 / 17$ \\
Cause of injury & & \\
$\quad$ Sports & 58 & 41 \\
Traffic accident & 9 & 10 \\
ADL or others & 4 & 5 \\
Associated lesion in same knee & & \\
$\quad$ Chondral lesion & 36 & 29 \\
$\quad$ Meniscal tear & 37 & 36 \\
$\quad$ MCL tear & 21 & 23 \\
Mean duration of follow-up (mo) & 22.7 & 14.5 \\
\hline
\end{tabular}

ADL: activities of daily living, MCL: medial collateral ligament.
The mean operation time was significantly different between the two groups with 58 minutes in group I (Rigid-fix group) and 69 minutes in group II (PINN ACL Cross Pin group) ( $\mathrm{p}=0.038$ ). The mean IKDC subjective score increased from 72.2 \pm 11.9 preoperatively to $94.1 \pm 6.8$ at the last follow-up in group I $(\mathrm{p}=0.036)$ and from $69.3 \pm 8.9$ preoperatively to $87 \pm 12.3$ at the last follow-up in group II ( $\mathrm{p}=0.028$ ), showing no statistically significant difference between the two groups. There was an improvement in the mean Lysholm score from 56.2 \pm 7.9 preoperatively to $95.4 \pm 6.8$ at the last follow-up in group I ( $\mathrm{p}=0.0231$ ) and from $64.6 \pm 11.9$ preoperatively to $91.2 \pm 12.1$ at the last follow-up in group II, but no significant difference was noted between the two groups. The mean laxity assessed using a KT1000 arthrometer improved from $6.39 \pm 2.9 \mathrm{~mm}$ preoperatively to $3.03 \pm 1.6 \mathrm{~mm}$ at the last follow-up in group $\mathrm{I}(\mathrm{p}=0.025)$ and from $6.68 \pm 3.3 \mathrm{~mm}$ preoperatively to $2.39 \pm 1.9 \mathrm{~mm}$ at the last follow-up, but no statistically significant difference between the two groups was observed. Also, improvement was observed in the mean side-to-side difference in anterior translation measured by Telos stress radiography from $7.65 \pm 2.0 \mathrm{~mm}$ preoperatively to $3.09 \pm 1.3 \mathrm{~mm}$ at the last follow-up in group I $(\mathrm{p}=0.015)$ and from $8.03 \pm 3.1 \mathrm{~mm}$ preoperatively to $2.55 \pm 1.9 \mathrm{~mm}$ at the last followup in group II ( $\mathrm{p}=0.031$ ); but no significant difference between the two groups was observed. Regarding the pivot shift test, there was an improvement in the integrity of the ACL in both the groups, but no significant difference was noted between the groups: there were 5 cases of grade I, 45 cases of grade II, and 21 cases of grade III preoperatively and 49 cases of grade 0,16 cases of grade I, and 6 cases of grade II at the last follow-up in group I ( $p=0.013$ ); whereas there were 6 cases of grade I, 37 cases of grade II, and 13 cases of grade III preoperatively and 38 cases of grade 0,12 cases of grade I, and 6 cases of grade II at the last followup in group II. A statistically significant difference between the groups was observed in the one-leg hop test with group I showing greater improvements than group II: there were 7 cases

Table 2. The Clinical and Radiologic Results of Rigid-fix and PINN-ACL Cross Pin for ACL Reconstruction using Hamstring Tendon

\begin{tabular}{lcccc}
\hline & Group 1 pre-op & Group 1 post-op & Group 2 pre-op & Group 2 post-op \\
\hline IKDC score & 72.2 & 94.1 & 69.3 & 87.3 \\
Lysholm' score & 56.2 & 95.4 & 64.6 & 91.2 \\
KT-1000 arthrometer $^{\mathrm{a}}$ ) & 6.39 & 3.03 & 6.68 & 2.39 \\
Telos stress arthrometer $^{\mathrm{b})}$ & 7.65 & 3.09 & 8.03 & 2.55 \\
Pivot shift test (grade 0/I/II/III) & $0 / 5 / 45 / 21$ & $49 / 16 / 6 / 0$ & $0 / 6 / 37 / 13$ & $38 / 12 / 6 / 0$ \\
One-leg hop test (grade 0/I/II/III) & $7 / 28 / 27 / 9$ & $35 / 28 / 6 / 2$ & $4 / 18 / 28 / 6$ & $19 / 26 / 7 / 4$ \\
\hline
\end{tabular}

IKDC score: International Knee Documentation Committee subjective score, ACL: anterior cruciate ligament.

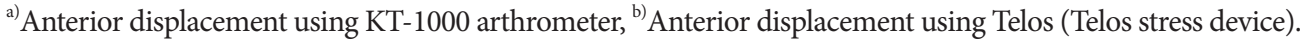


of grade 0,28 cases of grade I, 27 cases of grade II, and 9 cases of grade III preoperatively and 35 cases of grade 0,28 cases of grade I, 6 cases of grade II, and 2 cases of grade III at the last followup in group I ( $\mathrm{p}=0.032$ ); whereas there were 4 cases of grade 0 , 18 cases of grade I, 28 cases of grade II, and 6 cases of grade III preoperatively and 19 cases of grade 0,26 cases of grade I, 7 cases of grade II, and 4 cases of grade III at the last follow-up in group II $(\mathrm{p}=0.032)$ (Table 2).

Regarding the complications, the incidence of cross pin-femoral tunnel mismatch was higher in group II, with 4 cases in group I and 36 cases in group II ( $\mathrm{p}=0.017)$. Soft tissue irritation from cross pin head prominence was observed in 1 case in group I and 4 cases in group II, which further improved 2 months later in all the cases. No other complications including infection, fixation device failure, and fracture of the lateral femoral condyle were observed.

\section{Discussion}

The fixation mechanisms for femoral fixation of tendon grafts were classified by Milano et al. ${ }^{8}$ into compression, expansion, and suspension and the last one was subdivided into cortical suspension, cancellous suspension, and cortico-cancellous suspension. Cortical suspension fixation has been associated with biomechanical instability including bungee cord effect ${ }^{10)}$ and Windshield wiper effect ${ }^{11)}$. Compression fixation mechanism has a relatively low failure load and stability ${ }^{12,13)}$. Expansion fixation mechanism can be advantageous in obtaining secure fixation because two cross pins inserted transversely through a graft provides a centrifugal pressure on the femoral tunnel, but treatment results depend on the press-fit of the graft, bone density around the femoral tunnel, and correct placement of the cross pins through the graft tendon ${ }^{13-15)}$. In contrast, cancellous suspension offers secure fixation, stability, and stiffness because the length of the graft in the femoral tunnel is short with use of transverse fixation pins that can be inserted into the femoral metaphysis. Speirs et al. ${ }^{9)}$ reported the biomechanical superiority of the cancellous suspension fixation mechanism with respect to total creep, stability, and failure load. Accordingly, we attempted to assess the advantages and disadvantages of the PINNACL Cross Pin device, one of the devices used for cancellous suspension fixation by comparing it with the conventional Rigid-fix device. The clinical and radiological results of ACL reconstructions including early weight-bearing and rehabilitation were satisfying in both, group I (Rigid-fix group) and group II (PINN-ACL Cross Pin group). No significant difference could be observed between the groups except for the one-leg hop test. However, considering that greater clinical improvements were seen in group I, where the mean follow-up period was longer (group I: 22.7 months and group II: 14.5 months), a long-term follow-up study should be carried out for the better assessment of the efficacy of PINN-ACL Cross Pin device.

The incidence of cross pin-femoral tunnel mismatch was higher in group II with 36 cases. In our opinion, this was because the cross pin did not passed through the eyelet of the graft harness sliding in the femoral tunnel during cross pin drilling (Fig. 1). To prevent this, we tried to firmly fix the drill guide sheath to the femur or created a short femoral tunnel $(30-35 \mathrm{~mm})$ to perform drilling at almost perpendicular direction to the cortical bone. Soft tissue irritation from cross pin head prominence was observed in 4 cases. We hypothesize that this can be prevented by inserting cross pin heads more deeply or developing a smaller cross pin head.

\section{Conclusions}

In ACL reconstruction, Rigid-fix, a conventional fixation device, and PINN ACL Cross Pin, a recently introduced device, did not produce significantly different clinical and radiological results. However, the latter resulted in higher incidence of complications

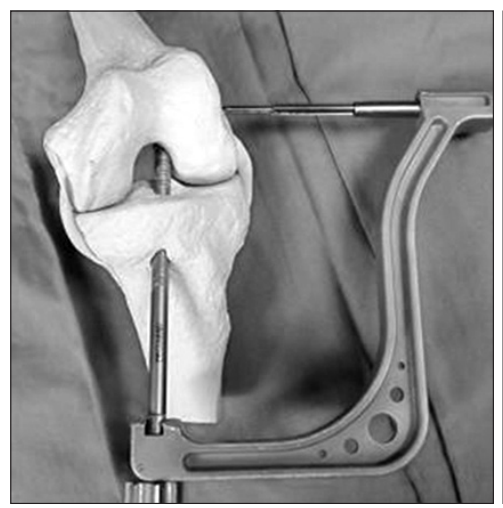

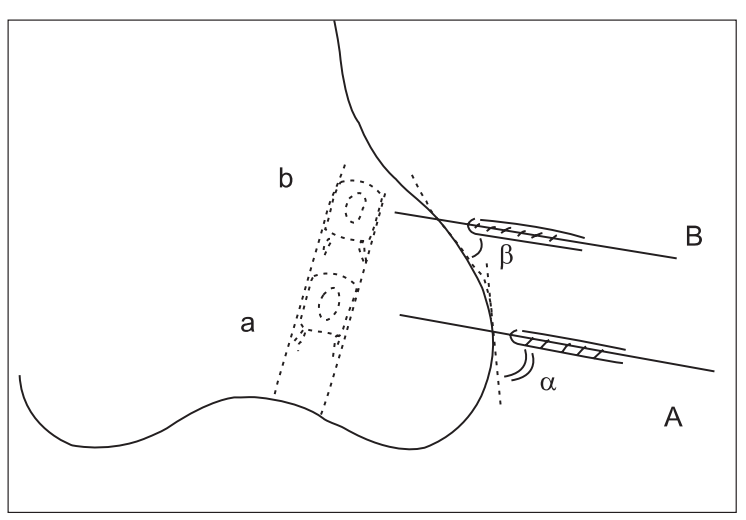

Fig. 1. Femoral tunnel-Cross pin and Graft Harness tunnel mismatch. When Graft Harness is positioned at ' $\mathrm{b}$, Cross pin tends to slide at the drilling site because it has a lower drill angle $(\beta<\alpha)$. 


\section{Seo et al. ACL Reconstruction with Autologous Hamstring Tendon}

including, cross pin-tunnel mismatch and required longer operation time. Therefore, we consider that further improvements in the device or surgical technique should be carried out. The limitations of this study include the short-term follow-up period and use of different tibial fixation methods between the two groups.

\section{References}

1. Ahn JH, Ha CW, Kim PS. Comparison of the clinical results of the fixation techniques to femur in ACL reconstruction using hamstring double-loops: bioscrews vs. semi-Fix. J Korean Knee Soc. 1999;11:32-8.

2. Amis AA. Anterior cruciate ligament replacement. Knee stability and the effects of implants. J Bone Joint Surg Br. 1989;71:819-24.

3. Corry IS, Webb JM, Clingeleffer AJ, Pinczewski LA. Arthroscopic reconstruction of the anterior cruciate ligament. A comparison of patellar tendon autograft and fourstrand hamstring tendon autograft. Am J Sports Med. 1999; 27:444-54.

4. Goldblatt JP, Fitzsimmons SE, Balk E, Richmond JC. Reconstruction of the anterior cruciate ligament: meta-analysis of patellar tendon versus hamstring tendon autograft. Arthroscopy. 2005;21:791-803.

5. Aune AK, Ekeland A, Cawley PW. Interference screw fixation of hamstring vs patellar tendon grafts for anterior cruciate ligament reconstruction. Knee Surg Sports Traumatol Arthrosc. 1998;6:99-102.

6. Brand J Jr, Weiler A, Caborn DN, Brown CH Jr, Johnson DL. Graft fixation in cruciate ligament reconstruction. Am J Sports Med. 2000;28:761-74.

7. Eriksson K, Anderberg P, Hamberg P, Lofgren AC, Bredenberg M, Westman I, Wredmark T. A comparison of quadruple semitendinosus and patellar tendon grafts in reconstruction of the anterior cruciate ligament. J Bone Joint Surg Br.
2001;83:348-54.

8. Milano G, Mulas PD, Ziranu F, Piras S, Manunta A, Fabbriciani C. Comparison between different femoral fixation devices for ACL reconstruction with doubled hamstring tendon graft: a biomechanical analysis. Arthroscopy. 2006;22:660-8.

9. Speirs A, Simon D, Lapner P. Evaluation of a new femoral fixation device in a simulated anterior cruciate ligament reconstruction. Arthroscopy. 2010;26:351-7.

10. Freedman KB, D’Amato MJ, Nedeff DD, Kaz A, Bach BR Jr. Arthroscopic anterior cruciate ligament reconstruction: a metaanalysis comparing patellar tendon and hamstring tendon autografts. Am J Sports Med. 2003;31:2-11.

11. Laxdal G, Kartus J, Hansson L, Heidvall M, Ejerhed L, Karlsson J. A prospective randomized comparison of bonepatellar tendon-bone and hamstring grafts for anterior cruciate ligament reconstruction. Arthroscopy. 2005;21:3442.

12. Charlton WP, Randolph DA Jr, Lemos S, Shields CL Jr. Clinical outcome of anterior cruciate ligament reconstruction with quadrupled hamstring tendon graft and bioabsorbable interference screw fixation. Am J Sports Med. 2003;31:518-21.

13. Kousa P, Jarvinen TL, Vihavainen M, Kannus P, Jarvinen M. The fixation strength of six hamstring tendon graft fixation devices in anterior cruciate ligament reconstruction. Part I: femoral site. Am J Sports Med. 2003;31:174-81.

14. Ahmad CS, Gardner TR, Groh M, Arnouk J, Levine WN. Mechanical properties of soft tissue femoral fixation devices for anterior cruciate ligament reconstruction. Am J Sports Med. 2004;32:635-40.

15. Zantop T, Weimann A, Rummler M, Hassenpflug J, Petersen W. Initial fixation strength of two bioabsorbable pins for the fixation of hamstring grafts compared to interference screw fixation: single cycle and cyclic loading. Am J Sports Med. 2004;32:641-9. 